\title{
Composição Corporal e Exigências Líquidas e Dietéticas de Macroelementos Inorgânicos (Ca, P, Mg, K e Na) de Bezerros da Raça Holandesa Alimentados com Dietas Contendo Diferentes Níveis de Volumoso ${ }^{1}$
}

\section{Ricardo Dias Signoretti ${ }^{2}$, José Fernando Coelho da Silva ${ }^{3}$, Sebastião de Campos Valadares Filho ${ }^{3}$, José Carlos Pereira ${ }^{3}$, Gherman Garcia Leal de Araújo ${ }^{5}$, Paulo Roberto Cecon ${ }^{4}$, Augusto César de Queiroz ${ }^{3}$}

\begin{abstract}
RESUMO - O objetivo deste trabalho foi avaliar os efeitos de diferentes níveis de volumoso nas dietas, sobre as exigências líquidas e dietéticas de cálcio $(\mathrm{Ca})$, fósforo $(\mathrm{P})$, magnésio $(\mathrm{Mg})$, potássio $(\mathrm{K})$ e sódio $(\mathrm{Na})$. Quarenta e quatro bezerros da raça Holandesa, puros por cruzamento, não-castrados, com idade média de 60 dias e peso vivo (PV) inicial de $78 \mathrm{~kg}$ foram usados. Oito animais foram abatidos ao início do experimento, como referência, para a estimativa do peso corporal vazio (PCVZ) inicial e das concentrações iniciais de Ca, $\mathrm{P}, \mathrm{Mg}, \mathrm{K}$ e Na no corpo. Os 36 animais restantes foram distribuídos nos tratamentos, em delineamento inteiramente casualizado, em quatro grupos de nove animais, de acordo com o nível de volumoso nas dietas: 10, 25, 40 e 55\%, na base da MS, usando-se feno de capim coast-cross (Cynodon dactylon), fubá de milho e farelo de soja, os quais constituíram dietas com aproximadamente 16\% de PB. Cinco animais de cada tratamento foram abatidos quando atingiram $190 \pm 10 \mathrm{~kg}$ PV e quatro quando atingiram $300 \pm 10 \mathrm{~kg}$ PV. Equações de regressão do logaritmo do conteúdo corporal de macroelementos inorgânicos, em função do logaritmo PCVZ, foram ajustadas para cada nível de volumoso. As exigências líquidas médias de $\mathrm{Ca}$ e $\mathrm{Mg}$ para ganho de $\mathrm{PCVZ}$ aumentaram, enquanto os requerimentos de $\mathrm{P}$, $\mathrm{K}$ e $\mathrm{Na}$ reduziram, com o PCVZ dos animais. As exigências líquidas de $\mathrm{Ca}, \mathrm{P}, \mathrm{Mg}, \mathrm{K}$ e $\mathrm{Na}$ para ganho de $1 \mathrm{~kg}$ de $\mathrm{PCVZ}$ para um animal de $300 \mathrm{~kg}$ PV, usando-se a equação geral, foram, respectivamente, 16,96; 9,59; 0,37; 1,88; e 1,20 g, enquanto as exigências dietéticas para ganho $1 \mathrm{~kg}$ de PV foram, respectivamente, 26,30; 17,40; 0,70;2,01; e 1,55 g. As concentrações corporais dos macroelementos inorgânicos foram influenciadas pelos níveis de volumoso e pelo aumento do PCVZ dos animais.
\end{abstract}

Palavras-chave: bezerro holandês, composição corporal, macroelementos inorgânicos, requerimentos

\section{Body Composition and Net and Dietary Inorganic Macroelements (Ca, P, Mg, K and Na) Requirements of Holstein Calves Fed Diets with Different Levels of Forage}

ABSTRACT - The objective of this work was to evaluate the effects of different forage levels in the diet on the net and dietary calcium $(\mathrm{Ca})$, phosphorus $(\mathrm{P})$, magnesium $(\mathrm{Mg})$, potassium $(\mathrm{K})$ and sodium $(\mathrm{Na})$ requirements. Forty-four pure Holstein bulls calves, 60 days old with initial average live weight ( $\mathrm{LW}$ ) of $78 \mathrm{~kg}$ were used. Eight reference animals were slaughtered at the beginning of the experiment, to estimate the initial empty body weight (EBW) and initial body concentrations of Ca, $\mathrm{P}, \mathrm{Mg}, \mathrm{K}$ and $\mathrm{Na}$. The remaining 36 animals were allotted in the treatments in a complete randomized experimental design, in four groups of nine animals, in function of forage levels in the diet: 10, 25, 40 and 55\%, on the DM basis, using chopped coast-cross (Cynodon dactylon) grass, ground corn and soybean meal, which constitute diets with approximately $16 \%$ CP. Five animals from each treatment were slaughtered with $190+10$ $\mathrm{kg} \mathrm{LW}$ and the other 4 with $300+10 \mathrm{~kg} \mathrm{LW}$. Regression equation of the logarithm of inorganic macrominerals body contents in function of the EBW logarithm, were fitted for each forage level. The average net requirements of $\mathrm{Ca}$ and $\mathrm{Mg}$ increased, while the requirements of $\mathrm{P}, \mathrm{K}$ and $\mathrm{Na}$ decreased with animal EBW. The net requirements of $\mathrm{Ca}, \mathrm{P}, \mathrm{Mg}, \mathrm{K}$ and $\mathrm{Na}$ for $1 \mathrm{~kg}$ EBW gain for an animal of $300 \mathrm{~kg}$ $\mathrm{LW}$, using the overall equation, were, respectively $16.96,9.59, .37,1.88$ and $1.20 \mathrm{~g}$, while the dietary requirements for $1 \mathrm{~kg} \mathrm{LW}$ gain were $26.30,17.40, .70,2.01$ and $1.55 \mathrm{~g}$, respectively. The body inorganic macroelements concentrations were affected by forage levels and by the increase of the EBW of the animals.

Key Words: Holstein calf, body composition, inorganic macroelements, requirements

\footnotetext{
${ }^{1}$ Parte da Tese apresentada à UFV, pelo primeiro autor como um dos requisitos para obtenção do Título de Doutor em Zootecnia. Área de concentração: Nutrição de Ruminantes.

2 Eng.-Agr., aluno de Doutorado em Zootecnia do DZO/UFV. Bolsista da CAPES.

3 Professor do DZO/UFV - 36571-000 - Viçosa, MG. Bolsista do CNPq.

4 Professor do Departamento de Matemática da UFV.

${ }^{5}$ Pesquisador do EMBRAPA - CPATSA - 56300-000 - Petrolina, PE.
} 


\section{Introdução}

Os elementos inorgânicos estão presentes em todos os tecidos dos animais vertebrados, em proporções e quantidades variáveis. No entanto, apesar de constituírem somente $4 \%$ do peso corporal de um animal, os minerais têm papel fundamental no metabolismo do animal, sendo essenciais tanto na utilização de proteína e energia, como na síntese de compostos essenciais ao organismo (DAYRELL, 1993).

A determinação dos requerimentos dos macroelementos inorgânicos para bovinos em crescimento é extremamente complexa, em virtude dos diversos fatores que influenciam a sua utilização pelo animal. Dentre os referidos fatores, os principais são: peso corporal, espécie ou raça, nível de produção, as inter-relações entre os minerais ou correlações entre as frações orgânica e inorgânica, a disponibilidade e a forma química do elemento nos ingredientes da dieta, o critério de estabelecimento de níveis adequados, o melhoramento contínuo das raças, o melhoramento de manejo e alimentação e os métodos de processamento dos alimentos (COELHO DA SILVA e LEÃO, 1979; ARC, 1980; MAYNARD et al., 1984; CONRAD et al., 1985; AFRC, 1991; BEEDE, 1991; e NRC, 1996).

O cálcio é o elemento inorgânico mais abundante do corpo animal, representando aproximadamente $2 \%$ do peso vivo do animal. Destes, em torno de $98 \%$ estão presentes no esqueleto e nos dentes e os $2 \%$ restantes, no fluido extracelular e nos tecidos moles do organismo (NRC, 1984, 1996).

A deposição de cálcio para ganho de peso varia amplamente com idade, nutrição prévia e taxa de crescimento do animal (COELHO DA SILVA, 1995). No entanto, o ARC (1980) considerou o valor de 14 $\mathrm{g} \mathrm{de} \mathrm{Ca} / \mathrm{kg}$ equivalente à composição do corpo vazio e do ganho de peso corporal vazio. Portanto, admitiuse que a deposição de cálcio no ganho é constante, independentemente da idade do animal.

Todavia, o AFRC (1991), reexaminando o modelo proposto pelo ARC (1980), introduziu o peso à maturidade em vez do PCVZ, considerando-se, dessa forma, decréscimo na deposição de cálcio com o avanço da maturidade. Foram estimadas as exigências líquidas de cálcio para bovinos em crescimento, considerando-se peso à maturidade de $900 \mathrm{~kg}$, em 16,$2 ; 13,9 ; \mathrm{e} 12,7 \mathrm{~g} \mathrm{de} \mathrm{Ca} / \mathrm{kg}$ de PV, respectivamente, para 100,200 e $300 \mathrm{~kg}$ de PV. A taxa de acúmulo dos minerais foi altamente influenciada pelo grau de maturidade e pela mineralização de animais abatidos mais jovens, sendo muito variável a mineralização por volta da desmama (AFRC,1991).

Os animais jovens absorvem $\mathrm{Ca}$ mais eficientemente que os adultos, porém, os requerimentos são maiores para os primeiros, em virtude da maior taxa de crescimento ósseo e das elevadas taxas de ganho (NRC, 1984).

O fósforo está distribuído no corpo animal de forma diferente da observada para o cálcio, constituindo $1 \%$ do peso corporal do animal, dos quais $80 \%$ estão no esqueleto e os $20 \%$ restantes, nos tecidos moles (COELHO DA SILVA, 1995).

O ARC (1980) considerou o valor de $8,0 \mathrm{~g}$ de $\mathrm{P} / \mathrm{kg}$ de PCVZ equivalente à composição do corpo $\mathrm{e}$ do ganho de peso corporal vazio, ou seja, admitiu que o depósito de fósforo no corpo é independente da idade. No entanto, de forma semelhante ao discutido para o cálcio, o AFRC (1991) também considerou que a deposição de fósforo no corpo do animal decresce, à medida que o animal se torna adulto. Dessa forma, foram estimadas as exigências líquidas de fósforo para o crescimento de bovinos, considerando-se peso à maturidade de $900 \mathrm{~kg}$, em 8,8; 7,7; e 7,2 g de P/kg de $\mathrm{PV}$, respectivamente, para 100, 200 e $300 \mathrm{~kg}$ de PV.

Em animais jovens, as necessidades dietéticas de cálcio são maiores que as de fósforo, mas tornam-se equilibradas, quando se aproxima a maturidade. A relação ideal Ca:P para bovinos em crescimento deve variar de 1:1 a 2:1, aproxima-se da existente entre os dois elementos inorgânicos nos ossos (CONRADetal., 1985).

Com relação ao conteúdo corporal de cálcio e fósforo do ganho de peso de bovinos da raça Holandesa, NOUR e THONNEY (1988) verificaram que a concentração corporal de cálcio foi maior em bovinos da raça Holandesa do que em Aberdeen Angus, atribuindo esta diferença a diferentes teores de gordura corporal, uma vez que bovinos da raça Holandesa apresentaram maior proporção de ossos no corpo que bovinos Aberdeen Angus.

Com aumento do teor de gordura corporal, geralmente, deveria haver queda nas concentrações dos elementos inorgânicos, pois os depósitos de gordura virtualmente não contêm cálcio, e sim quantidades mínimas de fósforo. Resultados de GALVÃO (1991), JORGE (1993) e PERON et al. (1993), referentes a animais abatidos com diferentes pesos vivos, mostraram acentuada redução da proporção de ossos na carcaça com a elevação do PVCZ, confirmando as quedas das concentrações de $\mathrm{Ca}$ e $\mathrm{P}$, uma vez que nos ossos se encontram os maiores depósitos dos dois elementos inorgânicos. 
Em revisão de literatura, NOUR e THONNEY (1988), quando verificaram a influência do conteúdo de gordura corporal sobre a concentração de minerais, concluíram que os animais com maior teor de gordura no corpo tendem a apresentar menor concentração de minerais na carcaça que animais magros.

O magnésio é o quarto cátion mais abundante no organismo animal, representando aproximadamente $0,05 \%$ do peso total e $0,5 \%$ da matéria mineral, dos quais em torno de $70 \%$ são encontrados no esqueleto, tornando-se assim, juntamente com o Ca e o P, fator essencial para a formação dos ossos e dentes. Encontram-se, também, 15\% de Mg nos músculos, cerca de 15\% em outros tecidos moles e 1\% no fluido extracelular. Este mineral desempenha, também, função essencial como ativador de diversas enzimas (NRC, 1996).

O ARC (1980) considerou, em conjunto, dados referentes a animais não-castrados, castrados e fêmeas, sugerindo conteúdo corporal e exigência líquida de $0,45 \mathrm{~g}$ de $\mathrm{Mg} / \mathrm{kg}$ de ganho, independente do PCVZ do animal.

$O$ requerimento dietético de magnésio varia conforme a idade, o estado fisiológico e a disponibilidade na dieta. A recomendação para bovinos em crescimento com base na MS é de $0,10 \%$ (NRC, 1996). Resultados médios de $0,50 \mathrm{~g}$ de $\mathrm{Mg} / \mathrm{kg}$ de PCVZ foram obtidos para bovinos da raça Holandesa, em experimentos realizados no Brasil (COELHO DA SILVA, 1995).

O potássio é o terceiro elemento inorgâncio mais abundante no organismo animal, participando, juntamente com o sódio, da manutenção do equilíbrio ácidobase e da pressão osmótica, na atividade de transmissão de impulsos nervosos, na contração muscular e de certas reações enzimáticas, entre outros (NRC, 1996).

O potássio está presente em ossos, músculos, tecidos nervosos, soro e outros fluidos do corpo, que contém, respectivamente, $50 \mathrm{mg} ; 4 \mathrm{~g} ; 3,5 \mathrm{~g}$; e $200 \mathrm{mg}$ de K/kg (COELHO DA SILVA, 1995).

O ARC (1980) considerou a concentração corporal e a exigência para ganho de peso de $2,0 \mathrm{~g}$ de $\mathrm{K} /$ $\mathrm{kg}$ de PCVZ. Em experimentos realizados no Brasil, para bovinos da raça Holandesa, o teor médio de potássio foi de 2,15 g/ $\mathrm{kg}$ de PCVZ (COELHO DA SILVA, 1995).

MEYER (1988) verificou que o conteúdo de $\mathrm{K}$ do ganho, geralmente, decresce com o aumento de peso, devido ao maior acúmulo de gordura e à menor quantidade de tecido muscular. Segundo WEIL et al. (1988) e TUCKER et al. (1991), o requerimento de K de bezerros da raça Holandesa, no início da fase de crescimento, foi de $0,55 \%$ na base da MS da dieta.
PIRES et al. (1991) encontraram, para bovinos com $200 \mathrm{~kg}$ de PCVZ, conteúdos de K no corpo de 2,17 e no ganho de 2,02 g/kg de GPCVZ.

O sódio é o principal cátion do fluido extracelular, como já foi mencionado, e participa juntamente com o potássio de diversas funções importantes no organismo animal.

O osso contém $4 \mathrm{~g}$ de $\mathrm{Na} / \mathrm{kg}$, os músculos são relativamente pobres em sódio, $750 \mathrm{mg} \mathrm{de} \mathrm{Na} / \mathrm{kg}$, e o fluido extracelular contém $3,5 \mathrm{~g}$ de Na/ $\mathrm{kg}$ (COELHO DA SILVA, 1995).

O ARC (1980) sugeriu valor de $1,5 \mathrm{~g}$ de $\mathrm{Na} / \mathrm{kg}$ de PCVZ, como requerimento líquido de sódio. O teor médio de sódio, em bovinos da raça Holandesa, foi de $1,81 \mathrm{~g} / \mathrm{kg}$ de PCVZ, obtido em estudo realizado no Brasil (COELHO DA SILVA, 1995).

CASTRO et al. (1993) estudaram a composição corporal e as exigências de macroelementos inorgânicos de bovinos de cinco grupos genéticos (14 meses de idade média e peso vivo inicial de $200 \mathrm{~kg}$ ). Esses autores verificaram que os conteúdos médios de $\mathrm{Ca}, \mathrm{P}, \mathrm{Mg}, \mathrm{K}$ e Na foram de 26,27; 12,83; 0,31; 1,9; e $1,99 \mathrm{~g} / \mathrm{kg}$ de ganho de peso vazio, respectivamente.

As estimativas das exigências dos macroelementos inorgânicos, para crescimento e engorda, têm sido obtidas pelo método fatorial. Para isso, há necessidade da obtenção das quantidades líquidas dos elementos inorgânicos depositados no corpo do animal, para atender ao crescimento e à engorda. A essas exigências líquidas são acrescidas as quantidades necessárias para atender às perdas inevitáveis do corpo, ou seja, as secreções endógenas, que são as exigências líquidas de manutenção. As frações de mantença e produção somadas constituem a exigência líquida total, que, corrigida por um coeficiente de absorção do elemento no trato gastrointestinal do animal, resulta na exigência dietética (ARC, 1980).

As informações sobre os requerimentos de macroelementos inorgânicos, publicadas no Brasil, para bovinos de corte (LANA, 1991; PIRES, 1991; SOARES, 1994; ESTRADA, 1996; PAULINO, 1996; e FERREIRA, 1997), dentre outros, e as para bovinos oriundos de rebanhos leiteiros (ARAÚJO, 1997) são ainda insuficientes para elaboração das normas brasileiras de exigências nutricionais.

O presente trabalho foi conduzido para avaliar o efeito de diferentes níveis de volumoso na dieta sobre a composição corporal e as exigências líquidas e dietéticas dos macroelementos inorgânicos $(\mathrm{Ca}, \mathrm{P}$, $\mathrm{Mg}, \mathrm{K}$ e $\mathrm{Na}$ ), de bezerros da raça Holandesa, nãocastrados, em confinamento. 


\section{Material e Métodos}

O local de condução do experimento, as dietas utilizadas, a proporção de ingredientes nas mesmas, o manejo e os animais utilizados, os ensaios de digestibilidade, os procedimentos de abate e as coletas de amostras de tecido animal e vegetal foram descritos em SIGNORETTI et al. (1999a,b).

Para determinação das exigências líquidas dos macroelementos inorgânicos $(\mathrm{Ca}, \mathrm{P}, \mathrm{Mg}, \mathrm{K}$ e Na), os animais foram submetidos inicialmente a um período de adaptação às dietas e instalações por 15 dias e, em seguida, a um período experimental variável para cada tratamento, até atingirem os pesos de abate pré-estabelecidos, como descrito por SIGNORETTI et al. (1999c).

A solução mineral para análise dos macroelementos inorgânicos nos tecidos animal foi preparada por via úmida. Após as devidas diluições, o fósforo foi determinado por colorimetria; o cálcio e o magnésio, por espectrofotometria de absorção atômica; e o potássio e o sódio, por espectrofotometria de chama (SILVA, 1990).

Os conteúdos corporais de macroelementos inorgânicos foram determinados em função de suas concentrações no couro, na cabeça, nos pés, no sangue, na cauda, nas vísceras, nos órgãos e nos componentes separados da seção HH (músculo, gordura e osso).

Para a predição dos conteúdos corporais de macroelementos inorgânicos retidos no corpo dos animais, adotou-se a equação de regressão do logaritmo da quantidade de cada elemento inorgânico, em função do logaritmo do peso corporal vazio (PCVZ), conforme o seguinte modelo:

em que

$$
\mathrm{Y}=\mu+\mathrm{bX}+\mathrm{e}
$$

$\mathrm{Y}=$ logaritmo do conteúdo total do macroelemento inorgânico $(\mathrm{kg})$ retido no corpo vazio;

$\mu=$ efeito de média (intercepto);

$\mathrm{b}=$ coeficiente de regressão do logaritmo do conteúdo do macroelemento inorgânico, em função do logaritmo do PCVZ;

$\mathrm{X}=$ logaritmo do PCVZ; e

$\mathrm{e}=$ erro aleatório.

Derivando-se as equações de predição do conteúdo do corporal de macroelementos inorgânicos, em função do logaritmo do PCVZ, obtiveram-se as equações de predição das exigências líquidas de cálcio, fósforo, magnésio, potássio e sódio para ganho de $1 \mathrm{~kg}$ de PCVZ, do tipo:

$$
\mathrm{Y}^{\prime}=\text { b. } 10^{\mathrm{a}} \cdot \mathrm{X}^{(\mathrm{b}-1)}
$$

em que
$\mathrm{Y}^{\prime}=$ exigêncialíquida de macroelementos inorgânicos (Ca, P, Mg, K e Na) para ganho de $1 \mathrm{~kg}$ PCVZ;

a e $b=$ intercepto e coeficiente de regressão, respectivamente, das equações de predição dos conteúdos corporais de macroelementos inorgânicos; e

$\mathrm{X}=\mathrm{PCVZ}$ em $\mathrm{kg}$.

Os requerimentos dietéticos dos macroelementos inorgânicos foram estimados utilizando-se as exigências líquidas de ganho específicas, para cada nível de volumoso, e estimados em conjunto para os grupos de $190 \pm$ $10 \mathrm{~kg}$ e $300 \pm 10 \mathrm{~kg}$, divididos pelos coeficientes médios de absorção aparente total, por nível de volumoso e média geral, obtidos por SIGNORETTI et al. (1999b).

Para determinação das exigências em função do peso vivo, foi utilizado o fator médio de 1,16 , obtido pela regressão do ganho de peso vivo, em função do ganho de PCVZ dos animais do presente estudo (SIGNORETTI et al., 1999c).

\section{Resultados e Discussão}

Derivando-se as equações de regressão, ajustadas para cada nível de volumoso nas dietas, bem como a equação geral, para todos os níveis, do logaritmo das quantidades de cálcio, fósforo e magnésio presentes no corpo vazio dos animais (grupos 1 e 2), em função do logaritmo do peso corporal vazio (PCVZ), foram obtidas as equações para estimativas dos conteúdos de cálcio, fósforo e magnésio por $\mathrm{kg}$ de ganho PCVZ (GPCVZ), para animais de 75 a $300 \mathrm{~kg}$ de peso vivo (PV) (Tabela 1).

Os resultados mostraram acréscimo na exigência líquida de cálcio, quando se utilizaram níveis de $10 \mathrm{e}$ $55 \%$ de volumoso na dieta e para a equação geral, enquanto para os níveis de 25 e $40 \%$ de volumoso houve decréscimo, com o aumento do peso do animal.

$\mathrm{O}$ aumento aparente do conteúdo de $\mathrm{Ca}$, com a elevação do PCVZ, para os níveis de 10 e 55\% de volumoso e para a equação geral, pode ser explicado, em parte, pela maior taxa de crescimento e pela proporção de ossos na carcaça nesta fase de crescimento dos animais, uma vez que o esqueleto contém cerca de $98 \%$ do cálcio corporal. Resultados semelhantes foram encontrados por ARAÚJO (1997).

O decréscimo aparente observado no conteúdo de $\mathrm{Ca}$, com a elevação do peso corporal, para animais alimentados com dietas contendo 25 e $40 \%$ de volumoso, são semelhantes aos obtidos por SOARES (1994), PAULINO (1996) e FERREIRA (1997). As equações do AFRC (1991) também estimaram menores concentrações de cálcio por kg de ganho, com a elevação do 
Rev. bras. zootec.

Tabela 1 - Exigências líquidas de cálcio, fósforo e magnésio $(\mathrm{g})$ para de ganho de peso corporal vazio e em conjunto (Geral), para os diferentes pesos vivos (PV) e pesos corporais vazios (PCVZ)

Table1 - Net requirements of calcium, phosphorus and magnesium $(\mathrm{g})$ for empty body weight gain and in overall for the different live weights (LW) and empty body weights (EBW)

\begin{tabular}{|c|c|c|c|c|c|c|}
\hline \multirow{3}{*}{$\begin{array}{l}\mathrm{PV}(\mathrm{kg}) \\
L W\end{array}$} & \multirow{3}{*}{$\begin{array}{l}\text { PCVZ }(\mathrm{kg}) \\
E B W\end{array}$} & \multicolumn{3}{|c|}{ Nível de volumoso (\%) } & \multirow[b]{3}{*}{55} & \multirow[b]{3}{*}{ Geral(Overall) } \\
\hline & & & orage le & & & \\
\hline & & 10 & 25 & 40 & & \\
\hline \multicolumn{7}{|c|}{ Cálcio (g/kg GPCVZ) } \\
\hline \multicolumn{7}{|c|}{ Calcium $(\mathrm{g} / \mathrm{kg} E B W G)$} \\
\hline 75 & 62,78 & 12,77 & 15,10 & 15,45 & 14,15 & 15,29 \\
\hline 100 & 84,77 & 14,43 & 14,66 & 13,59 & 16,46 & 15,63 \\
\hline 150 & 128,76 & 17,12 & 14,06 & 11,35 & 20,35 & 16,11 \\
\hline 200 & 172,74 & 19,31 & 13,66 & 10,00 & 23,61 & 16,46 \\
\hline 250 & 216,72 & 21,18 & 13,35 & 9,07 & 26,48 & 16,73 \\
\hline$\underline{300}$ & 260,70 & 22,85 & 13,11 & 8,38 & 29,08 & 16,96 \\
\hline \multicolumn{7}{|c|}{ Fósforo (g/kg GPCVZ) } \\
\hline \multicolumn{7}{|c|}{ Phosphorus (g/kg EBWG) } \\
\hline 75 & 62,78 & 10,18 & 9,72 & 9,80 & 9,54 & 10,19 \\
\hline 100 & 84,77 & 10,37 & 8,94 & 8,98 & 10,73 & 10,06 \\
\hline 150 & 128,76 & 10,63 & 7,96 & 7,95 & 12,62 & 9,88 \\
\hline 200 & 172,74 & 10,83 & 7,33 & 7,30 & 14,15 & 9,76 \\
\hline 250 & 216,72 & 10,97 & 6,88 & 6,83 & 15,45 & 9,66 \\
\hline 300 & 260,70 & 11,10 & 6,54 & 6,46 & 16,60 & 9,59 \\
\hline \multirow{2}{*}{\multicolumn{7}{|c|}{$\begin{array}{l}\text { Magnésio (g/kg GPCVZ) } \\
\text { Magnesium (g/kg EBWG) }\end{array}$}} \\
\hline & & & & & & \\
\hline 75 & 62,78 & 0,33 & 0,33 & 0,35 & 0,32 & 0,34 \\
\hline 100 & 84,77 & 0,34 & 0,34 & 0,32 & 0,36 & 0,35 \\
\hline 150 & 128,76 & 0,37 & 0,35 & 0,27 & 0,41 & 0,36 \\
\hline 200 & 172,74 & 0,39 & 0,35 & 0,25 & 0,45 & 0,36 \\
\hline 250 & 216,72 & 0,40 & 0,36 & 0,23 & 0,48 & 0,36 \\
\hline 300 & 260,70 & 0,41 & 0,36 & 0,21 & 0,51 & 0,37 \\
\hline
\end{tabular}

peso corporal. A possível explicação para esse fato, provavelmente, deve estar relacionada ao aumento do teor de gordura corporal e a redução na proporção de ossos na carcaça desses animais, à elevação do PCVZ.

Os resultados obtidos pela equação geral, para o conteúdo de cálcio no ganho de peso, para um animal de $300 \mathrm{~kg}$ de PV, foi de 16,96 g. Este valor é 7,89\% superior ao obtido por ARAÚJO (1997).

Utilizando-se o modelo matemático proposto pelo AFRC (1991), estimou-se a exigência líquida de cálcio para um bovino de $300 \mathrm{~kg}$ de $\mathrm{PV}$, considerando-se peso à maturidade de $500 \mathrm{~kg}$, em 11,0 g/kg de ganho. Este valor é $54,18 \%$ inferior ao valor encontrado no presente trabalho, contudo, quando comparado ao valor proposto pelo ARC (1980) de $14 \mathrm{~g} / \mathrm{kg}$ de ganho, é21,14\% inferior ao obtido neste trabalho $(16,96 \mathrm{~g})$.

As exigências líquidas de fósforo decresceram, quando os animais receberam rações contendo 25 e $40 \%$ de volumoso e para equação geral, enquanto para os níveis de 10 e $55 \%$ de volumoso houve acréscimo, com a elevação do peso corporal dos animais (Tabela 1). A explicação possível para o aumentona exigência líquidade $\mathrm{P}$, quando se utilizaram os referidos níveis de volumoso, com o aumento do $\mathrm{PCVZ}$, pode estar relacionada à maior proporção de ossos na carcaça, pois os animais estavam em fase inicial de crescimento e os ossos contêm cerca de $80 \%$ do P corporal.

O resultado obtido pela equação geral, para a exigência de fósforo no ganho de peso, para um animal de $300 \mathrm{~kg}$ de PV, foi de 9,59 g. Este valoré $12,69 \%$ superior ao obtido por ARAÚJO (1997).

Utilizando-se o modelo matemático proposto pelo AFRC (1991), para um bovino de $300 \mathrm{~kg}$ de PV, considerando-se peso à maturidade de $500 \mathrm{~kg}$, o requerimento líquido de fósforo por $\mathrm{kg}$ de ganho é de $6,39 \mathrm{~g}$, sendo $50,08 \%$ inferior ao valor $(9,59 \mathrm{~g})$ obtido no presente trabalho. Comparando-se ao valor de 8,0 $\mathrm{g}$ de $\mathrm{P} / \mathrm{kg}$ de ganho, proposto pelo ARC (1980), o valor encontrado neste trabalho é $19,88 \%$ superior.

Observou-se acréscimo na exigência líquida de $\mathrm{Mg}$, quando os animais receberam 10 e $55 \%$ de volumoso na ração, enquanto, para o nível de $40 \%$ de volumoso na dieta, decresceu com aumento do peso corporal. Contudo, para o nível de $25 \%$ de volumoso e para a equação geral, mostraram tendência de deposição constante de magnésio, independente do 
peso do animal (Tabela 1). A explicação para este fato, provavelmente, pode estar relacionada à maior proporção de ossos na carcaça desses animais na fase de crescimento estudado, pois em torno de $70 \%$ de $\mathrm{Mg}$ corporal estão presentes nos ossos.

Com base na equação geral, a exigência líquida de $\mathrm{Mg}$ para um animal de $300 \mathrm{~kg}$ de PV foi de $0,37 \mathrm{~g} /$ $\mathrm{kg}$ de PCVZ. Este valor é $8,82 \%$ superior ao obtido por ARAÚJO (1997). Em média, a exigência líquida de magnésio obtida no presente trabalho, com base na equação geral, é $21,62 \%$ inferior ao valor proposto pelo ARC (1980).

As exigências líquidas de potássio e sódio, por quilograma de ganho de PCVZ dos animais recebendo rações contendo níveis crescentes de volumoso, bem como estimativas em conjunto (Geral), para pesos vivos variando de 75 a $300 \mathrm{~kg}$, são apresentadas na Tabela 2 .

As exigências líquidas de potássio decresceram quando os níveis de volumoso foram de 10, 25 e 55\% e para equação geral, mas, quando os animais receberam rações com $40 \%$ de volumoso, a exigência aumentou, com a elevação do peso corporal dos animais.

O requerimento de potássio estimado pela equação geral, para um animal de $300 \mathrm{~kg}$ de PV (1,88 g/kg de GPCVZ), quando comparado ao valor obtido por ARAÚJO (1997) e ao proposto pelo ARC (1980), é 10,64 e 6,38\% inferior, respectivamente.

As exigências líquidas de sódio decresceram quando os animais foram alimentados com rações contendo 25 e $40 \%$ de volumoso, bem como para equação geral, todavia, para o nível de 55\%, aumentaram com a elevação do PCVZ dos animais. Para o nível de $10 \%$ de volumoso, mostraram tendência de deposição constante de sódio, independente do peso do animal.

A partir dos resultados obtidos pela equação geral, observou-se que a exigência líquida de sódio, para ganho de $1 \mathrm{~kg}$ de PCVZ, decresceu de 1,25 para 1,20 , quando se elevou o peso vivo de 75 para $300 \mathrm{~kg}$. Estes valores situam-se abaixo do recomendado pelo ARC (1980), que é de $1,5 \mathrm{~g}$ de $\mathrm{Na} / \mathrm{kg}$ de GPCVZ, e acima do obtido por ARAÚJO (1997), que é de $0,89 \mathrm{~g}$ de Na/kg de GPCVZ.

Considerando-se os dados referentes aos coeficientes médios de absorção aparente determinados por SIGNORETTI et al. (1999b), mostrados na Tabela 3, foram estimados os requerimentos dietéticos de $\mathrm{Ca}, \mathrm{P}$, $\mathrm{Mg}$, $\mathrm{K}$ e Na, por quilograma de ganho de peso corporal vazio. Utilizando-se o fator de correção de 1,16, para conversão das exigências para GPCVZ em ganho de peso vivo (GPV), determinado por SIGNORETTI et al. (1999c), foram determinadas as exigências para ganho de $1 \mathrm{~kg}$ de peso vivo, quando foram utilizados níveis crescentes de volumoso na dieta, bem como as estimativas tomadas em conjunto (Tabela 4).

O NRC (1989) recomenda, como requerimento de $\mathrm{Ca}$ e P, valores de 26 e $20 \mathrm{~g}$ /dia, respectivamente, para um bovino leiteiro em crescimento, não-castrado, com $300 \mathrm{~kg}$ de $\mathrm{PV}$ e ganhando $1 \mathrm{~kg}$ de PV/dia, porém, o sistema americano estimou esses requerimentos utilizando coeficientes de absorção real de 38 e 50\%,

Tabela 2 - Exigências líquidas de potássio e sódio (g) para ganho de peso corporal vazio e em conjunto (Geral), para os diferentes pesos vivos (PV) e pesos corporais vazios (PCVZ)

Table 2 - Net requirements of potassium and sodium (g) forempty body weight gain and in overall for the different live weights (LW) and empty body weights (EBW)

\begin{tabular}{|c|c|c|c|c|c|c|}
\hline \multirow[t]{2}{*}{$\begin{array}{l}\overline{\mathrm{PV}}(\mathrm{kg}) \\
L W\end{array}$} & \multirow[t]{2}{*}{$\begin{array}{c}\text { PCVZ (kg) } \\
E B W\end{array}$} & \multicolumn{4}{|c|}{ Nível de volumoso (\%) } & \multirow[b]{2}{*}{ Geral (Overall) } \\
\hline & & 10 & 25 & 40 & 55 & \\
\hline \multicolumn{7}{|c|}{ Potássio (g/kg GPCVZ) } \\
\hline \multicolumn{7}{|c|}{ Potassium (g/kg EBWG) } \\
\hline$\overline{75}$ & 62,78 & 1,93 & 1,98 & 1,97 & 2,04 & 1,99 \\
\hline 100 & 84,77 & 1,90 & 1,95 & 2,01 & 2,00 & 1,97 \\
\hline 150 & 128,76 & 1,86 & 1,90 & 2,05 & 1,94 & 1,93 \\
\hline 200 & 172,74 & 1,83 & 1,87 & 2,09 & 1,90 & 1,91 \\
\hline 250 & 216,72 & 1,80 & 1,85 & 2,11 & 1,87 & 1,90 \\
\hline 300 & 260,70 & 1,78 & 1,83 & 2,13 & 1,85 & 1,88 \\
\hline \multicolumn{7}{|c|}{ Sódio (g/kg GPCVZ) } \\
\hline \multicolumn{7}{|c|}{ Sodium $(\mathrm{g} / \mathrm{kg} E B W G)$} \\
\hline$\overline{75}$ & 62,78 & 1,21 & 1,24 & 1,28 & 1,27 & 1,25 \\
\hline 100 & 84,77 & 1,21 & 1,22 & 1,23 & 1,31 & 1,24 \\
\hline 150 & 128,76 & 1,20 & 1,18 & 1,17 & 1,37 & 1,22 \\
\hline 200 & 172,74 & 1,20 & 1,16 & 1,13 & 1,41 & 1,21 \\
\hline 250 & 216,72 & 1,20 & 1,14 & 1,10 & 1,45 & 1,20 \\
\hline 300 & 260,70 & 1,20 & 1,12 & 1,08 & 1,48 & 1,20 \\
\hline
\end{tabular}


Rev. bras. zootec.

Tabela 3 - Coeficientes médios de absorção aparente para os teores de cálcio $(\mathrm{Ca})$, fósforo $(\mathrm{P})$, magnésio $(\mathrm{Mg})$, potássio $(\mathrm{k})$ e sódio $(\mathrm{Na})$

Table 3 - Average coefficients of apparent absorption for the calcium (Ca), phosphorus $(P)$, magnesium $(\mathrm{Mg})$, potassium $(\mathrm{K})$ and sodiun $(\mathrm{Na})$ contents

\begin{tabular}{lccccc}
\hline & \multicolumn{5}{c}{$\begin{array}{c}\text { Nível de volumoso (\%) } \\
\text { Forage level }\end{array}$} \\
\cline { 2 - 5 } $\begin{array}{l}\text { Elemento } \\
\text { Element }\end{array}$ & 10 & 25 & 40 & 55 & $\begin{array}{c}\text { Média } \\
\text { Mean }\end{array}$ \\
\hline $\mathrm{Ca}$ & 62,21 & 55,15 & 51,17 & 55,73 & 56,07 \\
$\mathrm{P}$ & 53,82 & 47,93 & 41,03 & 48,91 & 47,92 \\
$\mathrm{Mg}$ & 48,69 & 45,52 & 47,92 & 42,06 & 46,05 \\
$\mathrm{~K}$ & 84,48 & 83,18 & 78,35 & 79,91 & 81,48 \\
$\mathrm{Na}$ & 75,44 & 66,35 & 63,23 & 64,50 & 67,38 \\
\hline
\end{tabular}

respectivamente, para cálcio e fósforo.

Os valores encontrados neste trabalho, considerando a equação geral, para um animal de $300 \mathrm{~kg}$ de PV, foram de 26,30 e 17,40 g para cálcio e fósforo, respectivamente (Tabela 4). Estes valores, quando comparados aos encontrados por ARAÚJO (1997) para bezerros mestiços Holandês x Zebu, são 17,11\% inferiores em relação ao Ca e 6,10\% superiores em relação ao $\mathrm{P}$.

As exigências dietéticas de magnésio, estimadas pela equação geral, variaram de 0,64 a $0,70 \mathrm{~g} / \mathrm{kg}$ de GPV, quando o peso vivo se elevou de 75 para $300 \mathrm{~kg}$ (Tabela 4). Ovalor encontrado neste trabalho, consideran-

Tabela 4 - Exigências dietéticas de cálcio, fósforo, magnésio, potássio e sódio, para ganho de peso vivo e em conjunto (Geral), para os diferentes pesos vivos (PV)

Table 4 - Dietary requirements of calcium (Ca), phosphorus $(P)$, magnesium $(\mathrm{Mg})$, potassium $(\mathrm{K})$ and sodiun (Na), for live weight gain and in overall for the different live weights (LW)

\begin{tabular}{|c|c|c|c|c|c|}
\hline \multirow{3}{*}{$\begin{array}{l}\mathrm{PV}(\mathrm{kg}) \\
L W\end{array}$} & \multicolumn{4}{|c|}{ Nível de volumoso (\%) } & \multirow[b]{3}{*}{ Geral (Overall) } \\
\hline & \multicolumn{4}{|c|}{ Forage level } & \\
\hline & 10 & 25 & 40 & 55 & \\
\hline & \multicolumn{5}{|c|}{ Cálcio (g/kg GPV) } \\
\hline & \multicolumn{5}{|c|}{ Calcium ( $g / k g L W G)$} \\
\hline$\overline{75}$ & 19,80 & 23,42 & 23,96 & 21,94 & 23,71 \\
\hline 100 & 22,38 & 22,74 & 21,08 & 25,53 & 24,24 \\
\hline 150 & 26,55 & 21,81 & 17,60 & 31,56 & 24,98 \\
\hline 200 & 29,95 & 21,18 & 15,51 & 36,62 & 25,53 \\
\hline 250 & 32,85 & 20,70 & 14,07 & 41,07 & 25,95 \\
\hline \multirow[t]{3}{*}{300} & 35,85 & 20,33 & 13,00 & 45,08 & 26,30 \\
\hline & \multicolumn{5}{|c|}{ Fósforo (g/kg GPV) } \\
\hline & \multicolumn{5}{|c|}{ Phosphorus (g/kg LWG) } \\
\hline$\overline{75}$ & 18,47 & 17,64 & 17,75 & 17,31 & 18,49 \\
\hline 100 & 18,82 & 16,22 & 16,30 & 19,47 & 18,26 \\
\hline 150 & 19,29 & 14,44 & 14,43 & 22,90 & 17,93 \\
\hline 200 & 19,25 & 13,30 & 13,25 & 25,68 & 17,71 \\
\hline 250 & 19,92 & 12,48 & 12,39 & 28,04 & 17,53 \\
\hline \multirow[t]{3}{*}{300} & 20,14 & 11,85 & 11,76 & 30,12 & 17,40 \\
\hline & \multicolumn{5}{|c|}{ Magnésio (g/kg GPV) } \\
\hline & \multicolumn{5}{|c|}{ Magnesium $(g / k g ~ L W G)$} \\
\hline$\overline{75}$ & 0,62 & 0,62 & 0,66 & 0,60 & 0,64 \\
\hline 100 & 0,64 & 0,64 & 0,60 & 0,68 & 0,66 \\
\hline 150 & 0,70 & 0,66 & 0,51 & 0,77 & 0,68 \\
\hline 200 & 0,74 & 0,66 & 0,47 & 0,85 & 0,68 \\
\hline 250 & 0,76 & 0,68 & 0,43 & 0,91 & 0,68 \\
\hline \multirow[t]{3}{*}{300} & 0,77 & 0,68 & 0,40 & 0,96 & 0,70 \\
\hline & \multicolumn{5}{|c|}{ Potássio (g/kg GPV) } \\
\hline & \multicolumn{5}{|c|}{ Potassium ( $(\mathrm{g} / \mathrm{kg} L W G)$} \\
\hline$\overline{75}$ & 2,06 & 2,11 & 2,10 & 2,18 & 2,12 \\
\hline 100 & 2,03 & 2,08 & 2,15 & 2,13 & 2,10 \\
\hline 150 & 1,99 & 2,03 & 2,19 & 2,07 & 2,06 \\
\hline 200 & 1,95 & 2,00 & 2,23 & 2,03 & 2,04 \\
\hline 250 & 1,92 & 1,97 & 2,25 & 2,00 & 2,03 \\
\hline \multirow[t]{3}{*}{300} & 1,90 & 1,95 & 2,27 & 1,97 & 2,01 \\
\hline & \multicolumn{5}{|c|}{ Sódio (g/kg GPV) } \\
\hline & \multicolumn{5}{|c|}{ Sodium $(g / k g L W G)$} \\
\hline$\overline{75}$ & 1,56 & 1,60 & 1,65 & 1,64 & 1,61 \\
\hline 100 & 1,56 & 1,57 & 1,59 & 1,69 & 1,60 \\
\hline 150 & 1,55 & 1,52 & 1,51 & 1,77 & 1,57 \\
\hline 200 & 1,55 & 1,50 & 1,46 & 1,82 & 1,56 \\
\hline 250 & 1,55 & 1,47 & 1,42 & 1,87 & 1,55 \\
\hline 300 & 1,55 & 1,45 & 1,39 & 1,91 & 1,55 \\
\hline
\end{tabular}


do a equação geral, para um animal de $300 \mathrm{~kg}$ de PV, é $5,71 \%$ inferior ao valor estimado por ARAÚJO (1997). A recomendação para bovinos em crescimento proposta pelo NRC (1996) foi de $0,10 \%$ de Mg na MS da dieta, incluídas as necessidades de mantença e ganho.

As exigências dietéticas de potássio, estimadas pela equação geral, variaram de 2,12 a 2,01 g/ kg de GPV, quando o peso vivo se elevou de 75 para $300 \mathrm{~kg}$ (Tabela 4). O resultado obtido pela equação geral, para um animal de $300 \mathrm{~kg}$ de PV, foi de 2,01 g/kg de GPV, o qual é 25,87\% inferior ao valor obtido por ARAÚJO (1997). Segundo TUCKER et al. (1991), o requerimento de potássio de bezerros da raça Holandesa, ao início da fase de crescimento, foi de $0,55 \%$ na MS da dieta.

As exigências dietéticas de sódio, estimadas pela equação geral, variaram de 1,61 a 1,55 g/kg de GPV, quando o peso vivo se elevou de 75 para $300 \mathrm{~kg}$ (Tabela 4). Estes valores, em média, são inferiores aos valores obtidos por ARAÚJO (1997).

\section{Conclusões}

As exigências líquidas, obtidas pela equação geral, para ganho de $1 \mathrm{~kg}$ de PCVZ, para bezerros da raça Holandesa com peso variando de 75 a $300 \mathrm{~kg}$, variaram de 15,29 a $16,96 \mathrm{~g}$ para o $\mathrm{Ca} ; 9,59$ a $10,19 \mathrm{~g}$ para o $\mathrm{P}$; 0,34 a 0,37 g para o $\mathrm{Mg} ; 1,88$ a 1,99 g para o K; e 1,20 a 1,25 g para o $\mathrm{Na}$.

As exigências dietéticas, obtidas pela equação geral, para ganho de $1 \mathrm{~kg}$ de $\mathrm{PV}$, para bezerros da raça Holandesa com peso variando de 75 a $300 \mathrm{~kg}$, variaram de 23,71 a 26,30 g para o $\mathrm{Ca} ; 17,40$ a $18,49 \mathrm{~g}$ para o $\mathrm{P}$; 0,64 a $0,70 \mathrm{~g}$ para o $\mathrm{Mg} ; 2,01$ a 2,12 g para o $\mathrm{K}$; e 1,55 a $1,61 \mathrm{~g}$ para o $\mathrm{Na}$.

\section{Referências Bibliográficas}

AGRICULTURAL AND FOOD RESEARCHCOUNCIL - AFRC. 1991. Technical commitee on responses to nutrients, Report 6. A reappraisal of the calcium and phosphorous requirements os sheep and cattle. Nutr. Abs. Rev., 61(9):576-612.

AGRICULTURAL RESEARCH COUNCIL - ARC. 1980. The nutrients requirements of ruminants livestock. London: Commonwealth Agricultural Bureaux. 351p.

ARAÚJO, G.G.L. Consumo, digestibilidade, desempenho, composição corporal e exigências nutricionais de bezerros alimentados com dietas contendo diferentes níveis de volumoso. Viçosa, MG:UFV, 1997. 107 p. Tese (Doutorado em Zootecnia) - Universidade Federal de Viçosa, 1997.

BEEDE, D.K. Mineral and water nutrition in: Dairy nutrition management. Vet. Clin. N. Amer., 7(2):373 - 390, 1991.

CASTRO, A.C.G, COELHO DA SILVA, J.F., VALADARES FILHO, S.C. 1993. Composição corporal e exigências nutricionais de macroelementos inorgânicos para bovinos. $R$.
Soc. Bras. Zootec., 22(2):360 - 371.

COELHO DA SILVA, J.F. 1995. Exigências de macroelementos inorgânicos para bovinos: o sistema ARC/AFRC e a experiência no Brasil. In: PEREIRA, J.C. (Ed). SIMPÓSIO INTERNACIONAL SOBRE EXIGÊNCIAS NUTRICIONAIS DE RUMINANTES, 1995, Viçosa, MG. Anais... Viçosa, MG: JARD. p.467-504.

COELHO DA SILVA, J.F., LEÃO, M.I. 1979. Fundamentos de nutrição dos ruminantes. Piracicaba:Livroceres. 380p.

CONRAD, J.H., McDOWELL, L.R., ELLIS, G.L. et al. 1985. Minerais para ruminantes em pastejo em regiões tropicais. Campo Grande, MS: EMBRAPA - CNPGC. 90p.

DAYRELL, M.S. 1993. Deficiências minerais em bovinos do Brasil. In: PEIXOTO, A.M. (Ed). Nutrição de bovinos - conceitos básicos e aplicados. Piracicaba, SP: FEALQ. p.451 - 472.

ESTRADA, L.H.C. Composição corporal e exigências de proteína, energia e macroelementos minerais ( $\mathrm{Ca}, \mathrm{P}, \mathrm{Mg}, \mathrm{Na}$ e $\mathrm{K}$ ), características de carcaça e desempenho do nelore e mestiços em confinamento. Viçosa, MG:UFV, 1996.129p. Tese(Doutorado em Zootecnia) - Universidade Federal de Viçosa, 1996.

FERREIRA, M.A. Desempenho, exigências nutricionais e eficiência de utilização da energia metabolizável para ganho de peso de bovinos $F_{1}$ Simental $x$ Nelore. Viçosa, MG: UFV, 1997. 97p. Tese (Doutorado em Zootecnia) - Universidade Federal de Viçosa, 1997.

GALVÃO, J.G.C. Estudo da eficiência nutritiva, características e composição física da carcaça de bovinos de três grupos raciais, abatidos em três estágios de maturidade. Viçosa, MG UFV, 1991. 59p. Dissertação (Mestrado em Zootecnia) - Universidade Federal de Viçosa, 1991.

JORGE, A.M. Ganho de peso, conversão alimentar e características de carcaça de bovinos e bubalinos. Viçosa, MG UFV, 1993. 97p. Dissertação (Mestrado em Zootecnia) - Universidade Federal de Viçosa, 1993.

LANA, R. de P. Composição corporal e exigências de energia e proteina e de macroelementos minerais ( $\mathrm{Ca}, \mathrm{P}, \mathrm{Mg}, \mathrm{Na}$ e K) de novilhos de cinco grupos raciais, em confinamento. Viçosa, MG:UFV, 1991. 134 p. Dissertação (Mestrado em Zootecnia) - Universidade Federal de Viçosa, 1991.

MAYNARD, L.A., LOOSLI, J.K., HINTZ, H.F. et al. 1984. Nutrição animal. 3. ed. Rio de Janeiro: Freitas Bastos. 736 p.

MEYER, H. 1988. Potassium requirements and potassium supply in domestics animals. Anim. Res. Develop., 126:7-15.

NATIONAL RESEARCHCOUNCIL-NRC. Nutrient requirements of dairy cattle. 6.ed. Washington, DC:1989, 157 p.

NATIONAL RESEARCH COUNCIL - NRC. 1996. Nutrient requirements of beef cattle. 7.ed. Washington, DC: National Academy Press. 242 p.

NOUR, A.Y.M., THONNEY, M.L. 1988. Minerals of carcass solft tissue and bone of serially slaughtered cattle as affected by biological type and management. J. Agric. Sci., 11(1):41 - 49.

PAULINO, M.F. Composição corporal e exigências de energia, proteina e macroelementos minerais ( $\mathrm{Ca}, \mathrm{P}, \mathrm{Mg}, \mathrm{Na}$ e $\mathrm{K}$ ), de bovinos não castrados de quatro raças zebuinas em confinamento. Viçosa, MG:UFV, 1996. 80 p. Tese (Doutorado em Zootecnia) - Universidade Federal de Viçosa, 1996.

PERON, A.J., FONTES, C.A.A., LANA, R.P. et al. 1993. Rendimento de carcaça e de seus cortes básicos e área corporal de bovinos de cinco grupos genéticos, submetidos a alimentação restrita e "ad libitum". R. Soc. Bras. Zootec., 22(2):239 - 247.

PIRES, C.C. Exigências de proteína, energia e de macroelementos minerais ( $\mathrm{Ca}, \mathrm{P}, \mathrm{Mg}$, $\mathrm{Na}$ e $\mathrm{K}$ ) de novilhos e de bovinos nãocastrados de três grupos genéticos. Viçosa, MG:UFV, 1991. 125p. Tese (Doutorado em Zootecnia) - Universidade Federal de Viçosa, 1991. 
Rev. bras. zootec.

SIGNORETTI, R.D., COELHO DA SILVA, J.F., VALADARES FILHO, S.C. et al. 1999a. Consumo e digestibilidade aparente em bezerros da raça Holandesa alimentados com dietas contendo diferentes níveis de volumoso. Rev.bras. zootec., 28(1):169-177.

SIGNORETTI, R.D., COELHO DA SILVA, J.F., VALADARES FILHO, S.C. et al. 1999b. Consumo e absorção aparente total de macroelementos inorgânicos $(\mathrm{Ca}, \mathrm{P}, \mathrm{Mg}, \mathrm{K}, \mathrm{eNa})$, em bezerros da raça Holandesa alimentados com dietas contendo diferentes níveis de volumoso. Rev. bras. zootec., 28(1):178-184.

SIGNORETTI, R.D., COELHO DA SILVA, J.F., VALADARES FILHO, S.C. et al. 1999c. Composição corporal e exigências líquidas de energia e de proteína de bezerros da raça Holandesa alimentados com dietas contendo diferentes níveis de volumoso. Rev. bras. zootec., 28(1):185-194.

SILVA, D.J. 1990. Análise de alimentos (Métodos químicos e biológicos). Viçosa, MG:UFV. 196p.
SOARES, J. E. Composição corporal e exigências de macroelementos minerais ( $\mathrm{Ca}, \mathrm{P}, \mathrm{Mg}$, Na e K) para ganho de peso em bovinos (zebuínos e mestiços), e bubalinos. Viçosa, MG:UFV, 1994. 77 p. Dissertação (Mestrado em Zootecnia) - Universidade Federal de Viçosa, 1994.

TUCKER, W.B., JACKSON, J.A., HOPKINS, D.M. et al. 1991. Influence of dietary sodium bicarbonate on the potassium metabolism of growing dairy calves. J. Dairy Sci., 74:2296-2302.

WEIL, A.B., TUCKER, W.B., HEMKEN, R.W. 1988. Potassium requirements of dairy calves. J. Dairy Sci., 71(7):1868 - 1872.

Recebido em: 08/04/98

Aceito em: 20/08/98 